\title{
A search for radio supernovae and supernova remnants in the region of NGC 1569's super star clusters
}

\author{
A. Greve ${ }^{1}$, A. Tarchi ${ }^{2,3}$, S. Hüttemeister ${ }^{4,5}$, R. de Grijs ${ }^{6}$, J. M. van der Hulst ${ }^{7}$, \\ S. T. Garrington ${ }^{8}$, and N. Neininger ${ }^{2}$
}

1 Institut de Radio Astronomie Millimétrique, 300 rue de la Piscine, 38406 St. Martin d'Hères, France

2 Astronomisches Institut der Universität Bonn, Auf dem Hügel 71, 53121 Bonn, Germany

3 Max-Planck-Institut für Radioastronomie, Auf dem Hügel 69, 53121 Bonn, Germany

4 Astronomisches Institut der Ruhr-Universität Bochum, Universitätsstr. 150, 44780 Bochum, Germany

5 Onsala Space Observatory, 43920 Onsala, Sweden

${ }^{6}$ Institute of Astronomy, University of Cambridge, Madingley Road, Cambridge CB3 0HA, UK

7 Kapteyn Astronomical Instituut, Postbus 800, 9700 AV Groningen, The Netherlands

8 Nuffield Radio Astronomy Laboratories, Jodrell Bank, Macclesfield Cheshire SK11 9DL, UK

Received 20 April 2001 / Accepted 22 October 2001

\begin{abstract}
We have used MERLIN, at 1.4 and $5 \mathrm{GHz}$, to search for radio supernovae (RSNe) and supernova remnants (SNRs) in the unobscured irregular dwarf galaxy NGC 1569, and in particular in the region of its super star clusters (SSCs) A and B. Throughout NGC 1569 we find some 5 RSNe and SNRs but the SSCs and their immediate surroundings are largely devoid of non-thermal radio sources. Even though many massive stars in the SSCs are expected to have exploded already, when compared with M 82 and its many SSCs the absence of RSNe and SNRs in and near A and B may seem plausible on statistical arguments. The absence of RSNe and SNRs in and near A and B may, however, also be due to a violent and turbulent outflow of stellar winds and supernova ejected material, which does not provide a quiescent environment for the development of SNRs within and near the SSCs.
\end{abstract}

Key words. galaxies: individual: NGC 1569 - galaxies: ISM

\section{Introduction}

For some $150 \mathrm{Myr}$, the Magellanic-type irregular galaxy NGC 1569 experienced a starburst of relatively low average star formation rate $\left(\sim 0.5 M_{\odot} \mathrm{yr}^{-1}\right)$, until approximately $10 \mathrm{Myr}$ ago when the starburst gradually ceased. Throughout the body of NGC 1569 the starburst has produced many young stars (Greggio et al. 1998; Aloisi et al. 2001) and a large number of star clusters (Hunter et al. 2000), together containing a total mass of $\sim 10^{8} M_{\odot}$. However, evidence for recent and locally very efficient star formation in NGC 1569 comes from the Super Star Clusters (SSCs) A and B (Ables 1971; Arp \& Sandage 1985; O'Connell et al. 1994) which are similar in size and mass to those found in NGC 1705 (Melnick et al. 1985), M 82 (O'Connell et al. 1995; de Grijs et al. 2001), and other amorphous, irregular, and interacting galaxies. The SSCs are sites where in a relatively small volume of $\$ 50 \mathrm{kpc}^{3}$ a large number of stars $\left(\sim 10^{6} M_{\odot}\right)$ have spontaneously formed. Many massive stars produced in the starburst, either in the body of NGC 1569 or in and near

Send offprint requests to: A. Greve, e-mail: greve@iram.fr the SSCs and intermediate-size clusters, already ended in supernova (SN) explosions which created bubbles and kpc-sized loops, an outflow of hot X-ray emitting gas, and a component of young synchrotron radiation (Waller 1991; Heckman et al. 1995; Israel \& de Bruyn 1988). We may therefore expect that NGC 1569 contains a few radio supernovae (RSNe) and supernova remnants (SNRs), in particular in the region of the SSCs and the intermediatesize clusters where higher than average star formation occurred only 5-10 Myr ago (Prada et al. 1994; O'Connell et al. 1994; Origlia et al. 2001) and where some star formation may still go on, although today the galaxy contains only a few $10^{6} M_{\odot}$ of locally concentrated molecular gas (Greve et al. 1996; Taylor et al. 1999). We have observed NGC 1569 with MERLIN at 1.4 and $5 \mathrm{GHz}$ in order to search for RSNe and SNRs.

\subsection{NGC 1569 compared to the prototype starburst galaxy M 82}

For an investigation of the region around $\mathbf{A}$ and $\mathbf{B}$, a comparison with the SSC and SNR population and 
environment in the prototype starburst galaxy M 82 is highly relevant, in particular regarding the issue whether the RSNe and SNRs in M 82 are associated with SSCs. The starburst in M 82 has produced a large number of SSCs of which $\sim 200$ are seen with the HST, both in the active starburst regions "M $82 \mathrm{~A}$ " and "C" (O'Connell et al. 1995; nomenclature from O'Connell \& Mangano 1978), and in the more ancient starburst region "B" just outside the centre (de Grijs et al. 2001). These SSCs are $\geq 5-50$ Myr old so that many massive stars have already ended in a SN explosion. Although we may expect that some of the approximately 40, resp. 50 RSNe and SNRs in M 82 detected with MERLIN (Muxlow et al. 1994) and the VLA (Huang et al. 1994) are associated with SSCs, none, or at most one, coincides with the SSCs seen with the HST (Golla et al. 1996). Similarly, none - or at most one - of the $\sim 10 \mathrm{H}_{\alpha}$-bright SNR candidates detected by de Grijs et al. (2000) in M 82 B coincides with either bright VLA $8.4 \mathrm{GHz}$ sources, or the optically bright, slightly evolved SSCs found in large numbers in this region (cf. de Grijs et al. 2001). A simple calculation shows that in a population of 100 star clusters of ages similar to those estimated for M $82 \mathrm{~A}$ and containing $10^{5}$ and $10^{6}$ stars, one would expect to detect between about 5 and $\sim 50$ type II SNRs at any given moment, assuming any reasonable range of initial mass functions. The question remains, therefore, why none of the optically-detected young compact star clusters show any evidence for the presence of SNRs.

The hypothesis brought forward by Golla et al. (1996) for the absence of RSNe and SNRs in and near SSCs suggests that the visible SSCs of M 82 are located in the foreground and outside appreciable concentrations of interstellar gas so that the SN explosions were unable to sweep up gas and form SNRs. They argue furthermore that there are 1500-3000 SSCs in M 82 and that the detected RSNe and SNRs are hidden behind dense layers of dust so that the associated SSCs are not seen. This argument has apparently gained support from the recent MERLIN observation (Wills et al. 1998) of $\mathrm{H}$ I absorption in the direction of many RSNe and SNRs in M 82, and from estimates by Mattila \& Meikle (2001) that the MERLIN-detected sources in M 82 are hidden behind dust of $\left\langle A_{V}\right\rangle=24$ $(\sigma \approx 9)$ mag extinction. Evidently, under this condition none of the associated SSCs would be visible.

Taking M82 as example, on statistical arguments we may expect not to find in NGC 1569 a short-lived RSN or a SNR in or near the SSCs. If indeed the $\sim 3 \times 10^{8} M_{\odot}$ produced in the starburst of M 82 (McLeod et al. 1993) is primarily concentrated in the predicted 1500-3000 SSCs, and if the 40-50 RSNe and SNRs observed today originated in or near SSCs, then at present at most every $1 / 50$ th to 1/100th SSC would be associated with a RSN and SNR. Adopting similar conditions for the environment of the SSCs in NGC 1569, the chance to observe a RSN or SNR in or near $\mathbf{A}$ and $\mathbf{B}$, and in and near the intermediatesize clusters, is extremely small. This comparison however does not consider the possibility that SNRs in a dense gas environment, such as in M 82, may develop differently than in a Magellanic-type galaxy with generally a small amount of gas, such as in NGC 1569. Evidence and arguments for different conditions in the interstellar medium in M 82 have for instance been advocated by Pedlar et al. (1999) and Chevalier \& Fransson (2001). Finally, we may also argue that the environment in and near SSCs and the intermediate-size clusters may be particularly hostile at least for the formation of SNRs. The matter ejected in a SN explosion in or near the clusters is quickly dispersed because of stellar winds, nearby SN explosions, and the strong gravitational field of the clusters.

Because of the smaller distance to NGC 1569 (2.2 Mpc; Israel 1988) than to M 82 (3.6 Mpc; cf. Freedman et al. 1994; Sakai \& Madore 1999), 1.4 GHz and 5 GHz MERLIN observations with a resolution of 200 mas $(\sim 2 \mathrm{pc})$ and 50 mas $(\sim 0.5 \mathrm{pc})$, respectively, are suitable for a search of RSNe and SNRs. The RSNe and SNRs detected in M 82 with MERLIN (Muxlow et al. 1994; Wills et al. 1997) and the VLA (Huang et al. 1994) are either unresolved or have diameters of up to $\sim 5 \mathrm{pc}(\sim 400$ mas); the flux densities measured at $1.4 \mathrm{GHz}$ and $5 \mathrm{GHz}$ are between $\sim 0.5 \mathrm{mJy}$ and $\sim 20$ mJy. Similar sizes and flux densities are expected for the RSNe and SNRs in NGC 1569, if present at all.

\section{Observations}

A field of $\sim 2^{\prime} \times 1^{\prime}$ centered on the main body of NGC 1569 was observed for 20 hours with MERLIN ( 6 antennae) on Jan. 31 and Feb. 1, 1999. The observing frequency was $4.994 \mathrm{GHz}(\lambda=6 \mathrm{~cm})$ with a bandwidth of $15 \mathrm{MHz}$ in both circular polarizations. The data were taken in spectral-line mode $(32 \times 1 \mathrm{MHz}$ channels). The QSOs $0552+398(6.4 \mathrm{Jy})$ and $0402+682(0.18 \mathrm{Jy})$ were used as flux density and phase calibrators.

NGC 1569 was also observed with MERLIN (7 antennae, including the Lovell telescope) at $1.412 \mathrm{GHz}(\lambda=$ $21 \mathrm{~cm})$ for 36 hours in Apr. $(9,10,11 \mathrm{th})$ and May $(3 \mathrm{rd})$ 1999 , with a bandwidth of $15 \mathrm{MHz}$ in both polarizations. The passband and relative gain of the antennae were determined from observations of QSO $0552+398$ (1.75 Jy); the phases were determined from observations of QSO $0402+682(0.15 \mathrm{Jy})$.

Images were produced with the AIPS tasks IMAGR and deconvolved with CLEAN (Clark 1980). Table 1 summarizes the details of these observations. At $1.4 \mathrm{GHz}$, the rms-noise $\left(\sigma_{\mathrm{n}}\right)$ in source-free fields is consistent with the expected thermal noise level; for unknown reasons, at $5 \mathrm{GHz}$ the rms-noise is some $40 \%$ too high as compared to other observations (see Tarchi et al. 2000). At $1.4 \mathrm{GHz}$ and $5 \mathrm{GHz}$, respectively, sources weaker than $\sim 0.07 \mathrm{mJy} /$ beam $\left(=3 \sigma_{\mathrm{n}}\right)$ and $\sim 0.21 \mathrm{mJy} /$ beam $\left(=3 \sigma_{\mathrm{n}}\right)$ are therefore not detected.

Since the Wardle telescope was not available for observations, the shortest baseline at $1.4 \mathrm{GHz}$ was $7.5 \mathrm{~km}$ (Darnhall-Lovell telescopes) so that the array was not sensitive to extended structures larger than $\theta_{\max }^{1.4 \mathrm{GHz}}=$ $5.8^{\prime \prime}$ (equivalent to $70 \mathrm{pc}$ ). At $5 \mathrm{GHz}$ the shortest baseline was $8.7 \mathrm{~km}$ (Darnhall-Mark2 telescopes) so that, 


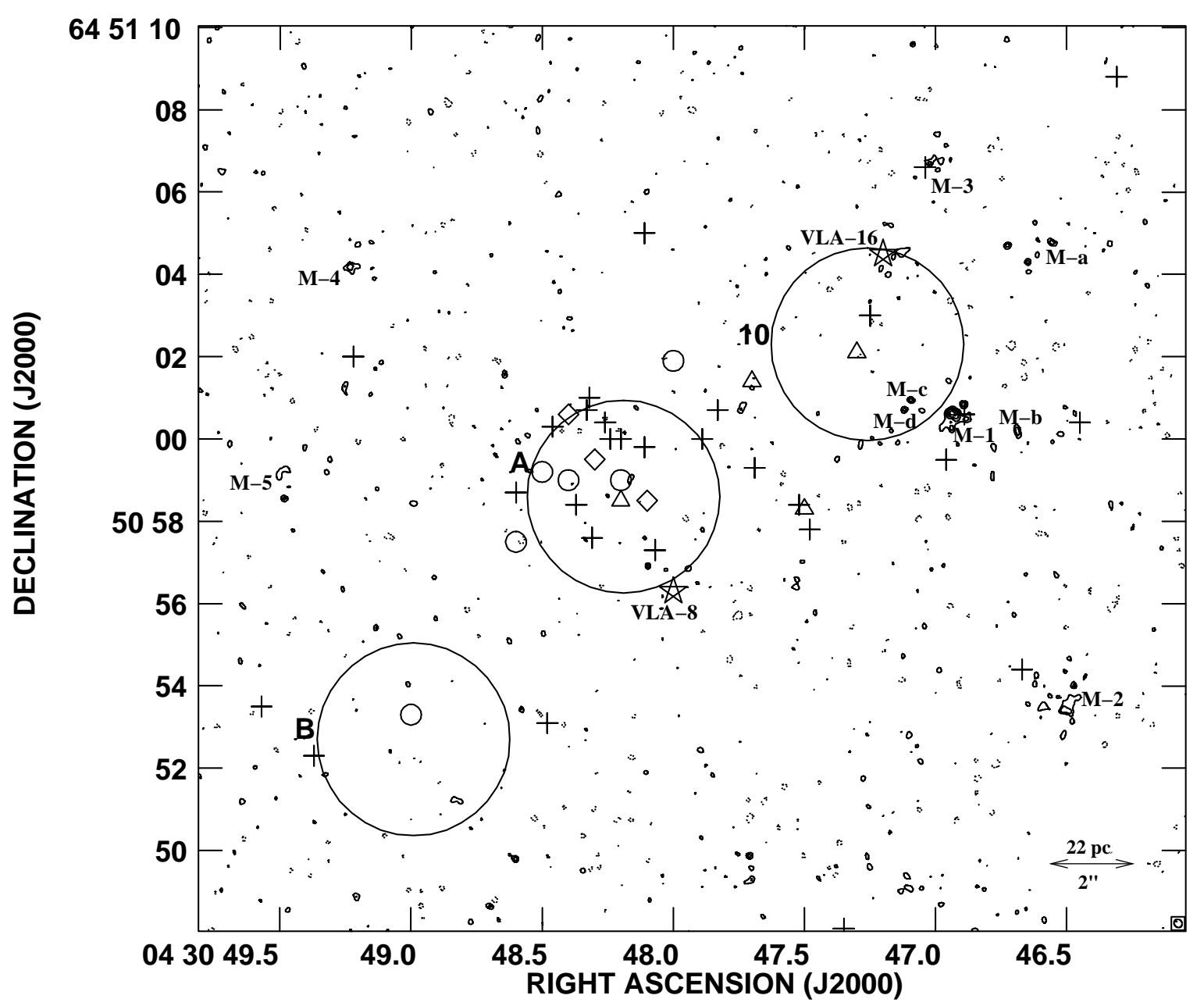

Fig. 1. 1.4 GHz MERLIN observation (contours in steps of $30 \mu \mathrm{Jy} /$ beam; first negative and positive contour at $50 \mu \mathrm{Jy} / \mathrm{beam}$ ) of the field around the super star clusters A and B and the star cluster C (= No. 10) of NGC 1569 (see Taylor et al. 1999 for a detailed $\mathrm{HST}, \mathrm{H}_{\alpha}$ and $\mathrm{CO}$ image). The encircled areas around the clusters have a diameter of $\sim 50 \mathrm{pc}$. The crosses show the positions of star clusters identified by Hunter et al. (2000); the open circles, diamonds, and triangles show the positions of WR objects (S, C, and U) observed by Buckalew et al. (2000). $1^{\prime \prime}$ is equivalent to $11 \mathrm{pc}$ at the distance of $2.2 \mathrm{Mpc}$. The MERLIN-detected sources M-1,2,3,4,5 (Table 2) and the tentative sources M-a, b, c, d (Table 3) are indicated. The stars show the positions of the VLA-detected sources No. 8 and No. 16 (vdHGI).

Table 1. MERLIN observations of NGC 1569; values for natural weighting.

\begin{tabular}{cccc}
\hline Frequency & $\begin{array}{c}\text { Beam } \\
\text { HPBW }\end{array}$ & $\begin{array}{c}\text { Beam at } \\
\text { NGC } 1569^{a}\end{array}$ & $\begin{array}{c}\text { rms-noise }\left(\sigma_{\mathrm{n}}\right) \\
{[\mu \mathrm{Jy} / \text { beam }]}\end{array}$ \\
\hline $1.4 \mathrm{GHz}$ & $0.21^{\prime \prime} \times 0.19^{\prime \prime}$ & $2.2 \mathrm{pc} \times 2.0 \mathrm{pc}$ & $\sim 25$ \\
$5 \mathrm{GHz}$ & $0.05^{\prime \prime} \times 0.05^{\prime \prime}$ & $0.5 \mathrm{pc} \times 0.5 \mathrm{pc}$ & $\sim 70$ \\
\hline
\end{tabular}

${ }^{a}$ For a distance of $2.2 \mathrm{Mpc}$.

similarly, $\theta_{\max }^{5 \mathrm{GHz}}=1.4^{\prime \prime}$ (equivalent to $17 \mathrm{pc}$ ). However, these fields are large enough for a search for RSNe, which appear point-like, and SNRs.

We compare the MERLIN observations with $20 \mathrm{~cm}$ and $6 \mathrm{~cm}$ VLA observations made on Sep. 24, 1982 (B-array: $20 \mathrm{~cm}$ and $6 \mathrm{~cm}$ ) and Nov. 22, 1983 (A-array: $20 \mathrm{~cm}$ ) by van der Hulst et al. (2001; abbreviated vdHGI). The radio images obtained with the VLA were made with $1.18^{\prime \prime}$ resolution (Gaussian beam) at both frequencies. The spectral indices $(\alpha)$ given below are derived from the VLA measurements.

At the distance of $2.2 \mathrm{Mpc}, 1^{\prime \prime}$ is equivalent to $11 \mathrm{pc}$ linear scale. The astrometric accuracy of the MERLIN observation is $\Delta \alpha=\Delta \delta \approx 0.01^{\prime \prime}$, of the VLA observation $\Delta \alpha=\Delta \delta \approx 0.05$ to $0.1^{\prime \prime}$.

We denote the MERLIN-detected sources by M, the VLA-detected soures (vdHGI) by VLA.

\section{The detected sources}

Throughout NGC 1569 we find at $1.4 \mathrm{GHz}$ six sources, M-1 to M-6 listed in Table 2, including their spectral indices $\alpha\left(S \propto \nu^{\alpha}, 1.4-5 \mathrm{GHz}\right)$. The sources are unresolved, except for the source M-6. Because of the (unexplained) high noise of the observations we do not detect any source above the $3 \sigma$ detection threshold at $5 \mathrm{GHz}$. The MERLIN 
Table 2. 1.4 GHz MERLIN-detected sources in NGC 1569.

\begin{tabular}{|c|c|c|c|c|c|c|c|}
\hline Source & $\begin{array}{c}\mathrm{RA}(2000) \\
{[\mathrm{h} \mathrm{m} \mathrm{s}]}\end{array}$ & $\begin{array}{c}\operatorname{Dec}(2000) \\
{\left[\begin{array}{lll}0 & \prime & \prime \prime\end{array}\right]}\end{array}$ & $\begin{array}{c}\text { Peak Flux } \\
{[\mathrm{mJy} / \text { beam }]}\end{array}$ & $\begin{array}{c}\text { Integr. Flux } \\
{[\mathrm{mJy}]}\end{array}$ & $\begin{array}{c}\text { Peak Flux }^{a} \\
{[\mathrm{mJy}]}\end{array}$ & $\begin{array}{r}\text { Spectral } \\
\text { Index }(\alpha)^{b}\end{array}$ & \\
\hline M-1 & 43046.94 & 645100.6 & $0.16(0.02)\left(6 \sigma_{\mathrm{n}}\right)$ & $0.57(0.20)$ & $1.353 \pm 0.039$ & $-0.02 \pm 0.05$ & \\
\hline M-2 & 4304 & 645053.4 & $12(0.02)\left(5 \sigma_{\mathrm{n}}\right)$ & $0.45(0.25)$ & $.848 \pm 0$ & $-0.56=$ & non- \\
\hline M-3 & 43047.02 & 645106.7 & $0.10(0.02)\left(4 \sigma_{\mathrm{n}}\right)$ & $0.10(0.04)$ & 1.107 & $-0.58=$ & non- \\
\hline M-4 & 43049.20 & 645104.3 & $0.11(0.02)\left(4 \sigma_{\mathrm{n}}\right)$ & $0.11(0.04)$ & $0.411 \pm 0.028$ & $0.04 \pm$ & Waller No. 5 \\
\hline M-5 & 43049.50 & 645059.3 & $0.09(0.02)\left(4 \sigma_{\mathrm{n}}\right)$ & $0.09(0.04)$ & $0.232 \pm 0.016$ & $-0.02 \pm 0.13$ & thermal \\
\hline$M-6$ & 43054.13 & 645043.5 & $0.19(0.02)\left(8 \sigma_{\mathrm{n}}\right)$ & $1.54(03.0)$ & $1.888 \pm 0.027$ & $-0.56 \pm 0.03$ & non-thermal \\
\hline
\end{tabular}

${ }^{a}$ VLA observation (van der Hulst et al. 2001), ${ }^{b} S \propto \nu^{\alpha}, 1.4-5 \mathrm{GHz},{ }^{c} \mathrm{H}$ II region number from Waller (1991); thermal source.

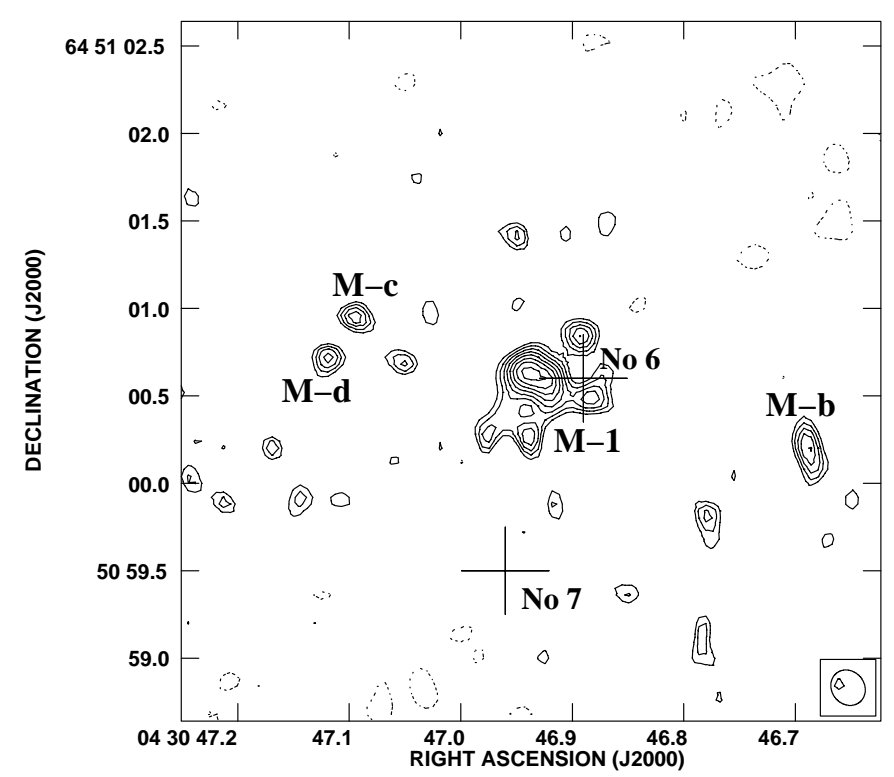

Fig. 2. 1.4 GHz MERLIN source M-1 (Table 2). The contour interval is $20 \mu \mathrm{Jy} /$ beam with the first negative and positive contour at $50 \mu \mathrm{Jy} /$ beam $\left(=2 \sigma_{\mathrm{n}}\right)$. The synthesized beam is shown in the lower right corner. The crosses indicate the center position of the star clusters No. 6 , at $\sim 3$ pc to the West, and No. 7 , at $\sim 12$ pc to the South (Table 4 ). The tentative sources $\mathrm{M}-\mathrm{b}, \mathrm{c}, \mathrm{d}$ (Table 3) are indicated.

sources detected at $1.4 \mathrm{GHz}$ are also present in the VLA observations of vdHGI.

Table 3 gives the positions where point-like emission of $\sim 0.1 \mathrm{mJy} /$ beam peak intensity $\left(\approx 4 \sigma_{\mathrm{n}}\right)$ is observed with MERLIN at $1.4 \mathrm{GHz}$. Without further observations we are unable to determine whether or not this emission is real, and associated with an otherwise identified source. However, at positions M-b, c, d the VLA (vdHGI) detects extended emission at a level of $0.7 \mathrm{mJy} /$ beam extending $2^{\prime \prime}$ to the west (possibly containing $\mathrm{M}-\mathrm{b}$ ) and $2^{\prime \prime}$ to the east (possibly containing M-c,d).

The MERLIN-detected sources are shown in Fig. 1; detailed images are shown in Figs. 2 to 7 . The stellar clusters located in the surroundings of the sources are listed in Table 4 below. In Figs. 2 to 7 the clusters are identified by the number in the list of Hunter et al. (2000).

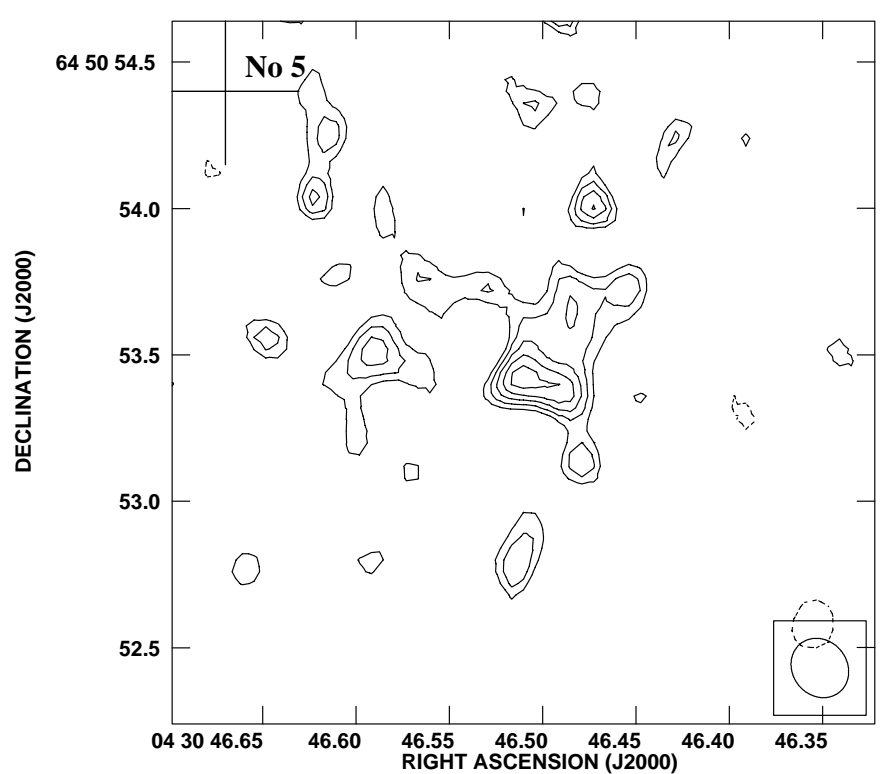

Fig. 3. 1.4 GHz MERLIN source M-2 (Table 2). The contour interval is $20 \mu \mathrm{Jy} /$ beam with the first negative and positive contour at $50 \mu \mathrm{Jy} /$ beam $\left(=2 \sigma_{\mathrm{n}}\right)$. The synthesized beam is shown in the lower right corner. The cross indicates the center position of the star cluster No. $5, \sim 15 \mathrm{pc}$ to the North-East (Table 4).

Table 3. Tentative $1.4 \mathrm{GHz}$ detections with MERLIN.

\begin{tabular}{cccc}
\hline Source & $\begin{array}{c}\text { RA (2000) } \\
{[\mathrm{h} \mathrm{m} \mathrm{s}]}\end{array}$ & $\begin{array}{c}\text { Dec (2000) } \\
{\left[{ }^{\circ} \text { ' }\right.}\end{array}$ & Figure \\
\hline $\mathbf{M}-\mathbf{a}$ & 43046.64 & 64514.7 & 1 \\
$\mathbf{M}-\mathbf{b}$ & 43046.68 & 64510.1 & 1,2 \\
$\mathbf{M}-\mathbf{c}$ & 43047.08 & 64510.9 & 1,2 \\
$\mathbf{M}-\mathbf{d}$ & 43047.11 & 64510.7 & 1,2 \\
\hline
\end{tabular}

We will now discuss the sources relevant to our understanding of the conditions in the galaxy's interstellar medium in more detail.

\subsection{Sources in the environment of SSC $A$ and $B$}

VLA-8 (Fig. 1): this non-thermal radio source with $\alpha=-0.24 \pm 0.10$ is found at $\sim 30 \mathrm{pc}$ to the South-West of 


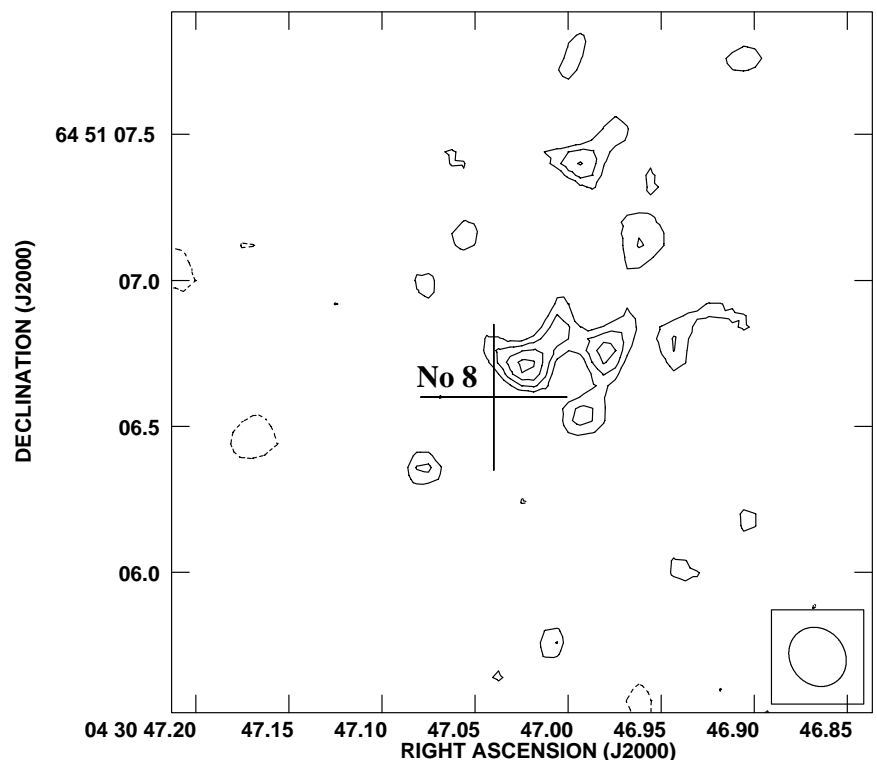

Fig. 4. 1.4 GHz MERLIN source M-3 (Table 2). The contour interval is $20 \mu \mathrm{Jy} /$ beam with the first negative and positive contour at $50 \mu \mathrm{Jy} /$ beam $\left(=2 \sigma_{\mathrm{n}}\right)$. The synthesized beam is shown in the lower right corner. The cross indicates the center position of the star cluster No. $8, \sim 2 \mathrm{pc}$ to the South-East (Table 4).

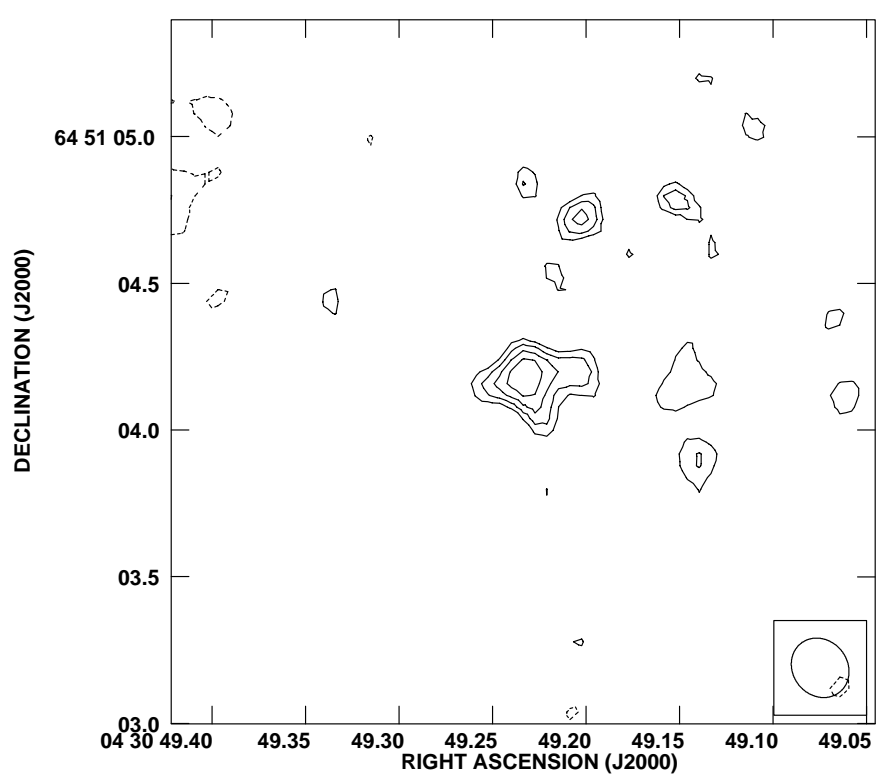

Fig. 5. $1.4 \mathrm{GHz}$ MERLIN source $\mathrm{M}-4$ (Table 2). The contour interval is $20 \mu \mathrm{Jy} /$ beam with the first negative and positive contour at $50 \mu \mathrm{Jy} /$ beam $\left(=2 \sigma_{\mathrm{n}}\right)$. The synthesized beam is shown in the lower right corner.

SSC-A at RA $04^{\mathrm{h}} 30^{\mathrm{m}} 48.0^{\mathrm{s}}$, Dec $64^{\circ} 50^{\prime} 56.3^{\prime \prime}$ (J2000). The peak flux densities are $S(1.4 \mathrm{GHz})=0.479 \pm 0.032 \mathrm{mJy}$, and $S(5 \mathrm{GHz})=0.357 \pm 0.022 \mathrm{mJy}$. The source is probably extended $(\sim 20 \mathrm{pc})$ and of low surface brightness since it is not seen with MERLIN; it may be a SNR based on its spectral appearance and extent (ruling out His regions and RSNe, respectively). The stellar cluster No. 18 is located

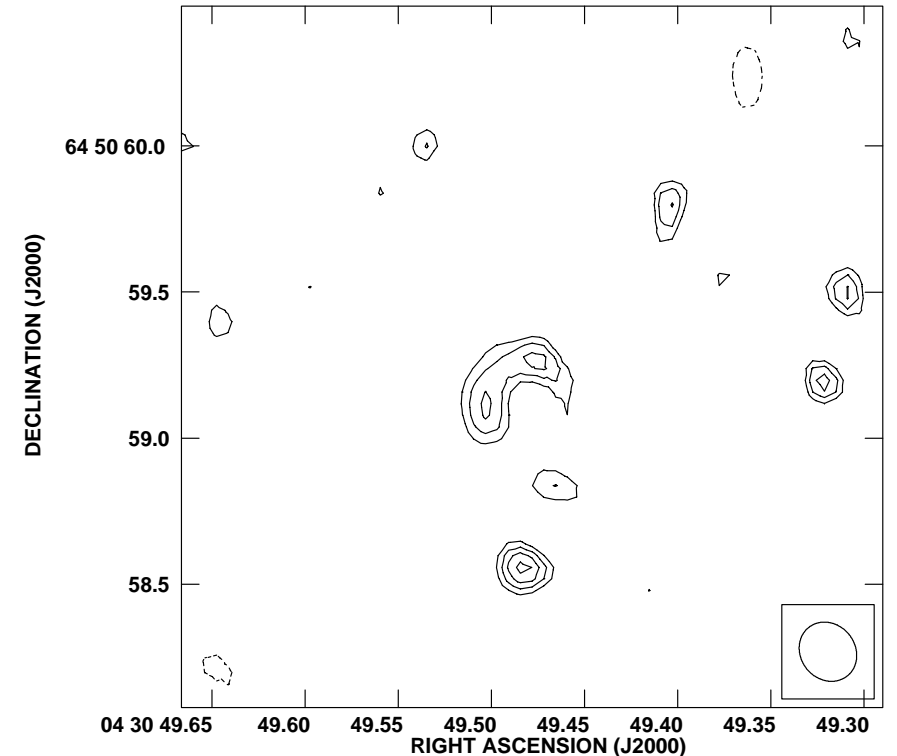

Fig. 6. 1.4 GHz MERLIN source M-5 (Table 2). The contour interval is $20 \mu \mathrm{Jy} /$ beam with the first negative and positive contour at $50 \mu \mathrm{Jy} /$ beam $\left(=2 \sigma_{\mathrm{n}}\right)$. The synthesized beam is shown in the lower right corner.

at $\sim 10 \mathrm{pc}$ to the North of the source (Table 4 ). In a region of $2^{\prime \prime} \times 2^{\prime \prime}(22 \mathrm{pc} \times 22 \mathrm{pc})$ centered on SSC- $\mathbf{A}$, Heckman et al. (1995) find $\mathrm{H}_{\alpha}$ emission of $1400-2300 \mathrm{~km} \mathrm{~s}^{-1}$ zerofull-width velocity which may be evidence of a Balmerdominated SNR. In view of the positional uncertainties, it is unclear whether both observations refer to the same object.

\subsection{Sources near cluster $C$ and the $C O$ cloud}

VLA-16 (Fig. 1): this source is found $\sim 25$ pc to the North of cluster C (No. 10 of Hunter et al. 2000; Table 4). The source is located at RA $4^{\mathrm{h}} 30^{\mathrm{m}} 47.2^{\mathrm{s}}$, Dec $64^{\circ} 51^{\prime} 04.5^{\prime \prime}$ (J2000). It has flux densities of $S(1.4 \mathrm{GHz})=0.681 \pm$ $0.046 \mathrm{mJy}$, and $S(5 \mathrm{GHz})=0.494 \pm 0.049 \mathrm{mJy}$, and a non-thermal spectral index of $\alpha=-0.27 \pm 0.14$. This source, not seen with MERLIN, could also be an extended ( 15 pc) low surface brightness SNR.

M-1 (Fig. 2): when compared with the HST, $\mathrm{H}_{\alpha}, \mathrm{CO}$ and star cluster images shown by Taylor et al. (1999) and Hunter et al. (2000), this source lies within, respectively, $3 \mathrm{pc}$ and $12 \mathrm{pc}$ distance from the clusters No. 6 and No. 7 (Hunter et al. 2000; Table 4). This source is located at the North-Eastern edge of a large molecular cloud complex (No. 3, 2, 1 in Taylor et al. 1999) and coincides with the H II region No. 2 of Waller (1991). The source is observed with the VLA (VLA-19) and has a thermal spectral index. M-1 may be double and a second source (cf. Fig. 2) may exist just to the North of the central stellar cluster No. 6 of Hunter et al. (2000). In this area of the extended H II region No. 2 (Waller 1991) lie also the tentative sources M-b, c, d (Table 3). 
M-2 (Fig. 3): this source is located at the South-Eastern side just outside the large molecular cloud complex $(3,2,1)$ of Taylor et al. (1999). The source lies at a distance of $\sim 15$ pc from the cluster No. 5 (Hunter et al. 2000; Table 4). The source was detected with the VLA (VLA-10) and has a non-thermal spectral index. It is most likely a RSN or a small SNR.

VLA-11: this non-thermal source with $\alpha=-0.74 \pm$ 0.09 is located some $\sim 100 \mathrm{pc}$ South of $\mathbf{C}$ and at the South-Western side just outside the molecular cloud complex $(3,2,1)$ of Taylor et al. (1999) at RA $4^{\mathrm{h}} 30^{\mathrm{m}} 45.79^{\mathrm{s}}$, Dec $64^{\circ} 50^{\prime} 58.3^{\prime \prime}$ (J2000), and with flux densities $S(1.4 \mathrm{GHz})=0.541 \pm 0.019 \mathrm{mJy}, S(5 \mathrm{GHz})=0.223 \pm$ $0.018 \mathrm{mJy}$. This source is not detected with MERLIN.

\subsection{Other sources}

M-3 (Fig. 4): this source is located $\sim 2$ pc to the West of cluster No. 8 of Hunter et al. (2000; Table 4). The source is also detected by the VLA (VLA-15). It has a non-thermal spectral index and is most likely a RSN or small SNR.

M-4, 5 (Figs. 5, 6): the source M-4 (VLA-7) is a thermal source close to the HiI region No. 5 (Waller 1991); the source M-5 (VLA-6) is a non-thermal source with some thermal emission.

M-6 (Fig. 7): this source lies in the area of the H II regions 6,7 and 9 identified by Waller (1991), but does not coincide with any of these objects nor with the H II complex observed by Seaquist et al. (1976). The MERLIN $1.4 \mathrm{GHz}$ observation shows an extended source of $\sim 17 \mathrm{pc}$ diameter. The source is detected with the VLA (VLA-1) and has a non-thermal spectrum $(\alpha=-0.55 \pm 0.02)$. In this area a SNR is detected based on [FeII] line emission (Labrie \& Pritchet 1998), however, the astrometric precision of this observation is not sufficient to confidently establish full correspondence.

Approximately $500 \mathrm{pc}$ to the East of $\mathbf{A}$ and $\mathbf{B}$, the VLA observations reveal 3 to 4 non-thermal sources (VLA-2, 3, 4, 5; $S(1.4 \mathrm{GHz}) \lesssim 0.4 \mathrm{mJy})$ in the H II region complex at $\mathrm{RA} \approx 4^{\mathrm{h}} 30^{\mathrm{m}} 52^{\mathrm{s}}$, Dec $64^{\circ} 50^{\prime} 45^{\prime \prime}$ (J2000) (see Seaquist et al. 1976) which are not seen by MERLIN. If extended, these sources are certainly below the detection limit of MERLIN.

\section{Discussion}

Throughout NGC 1569 we find 4 to 5 RSNe and/or SNRs, located within an area of $\sim 300 \mathrm{pc}$ diameter around the clusters $\mathbf{A}, \mathbf{B}, \mathbf{C}$ where active star formation occurred until recently, and might still be going on. We discuss separately the region near cluster $\mathbf{C}$ and the associated molecular cloud, and the SSCs A, B and the intermediate-size clusters and their surroundings.

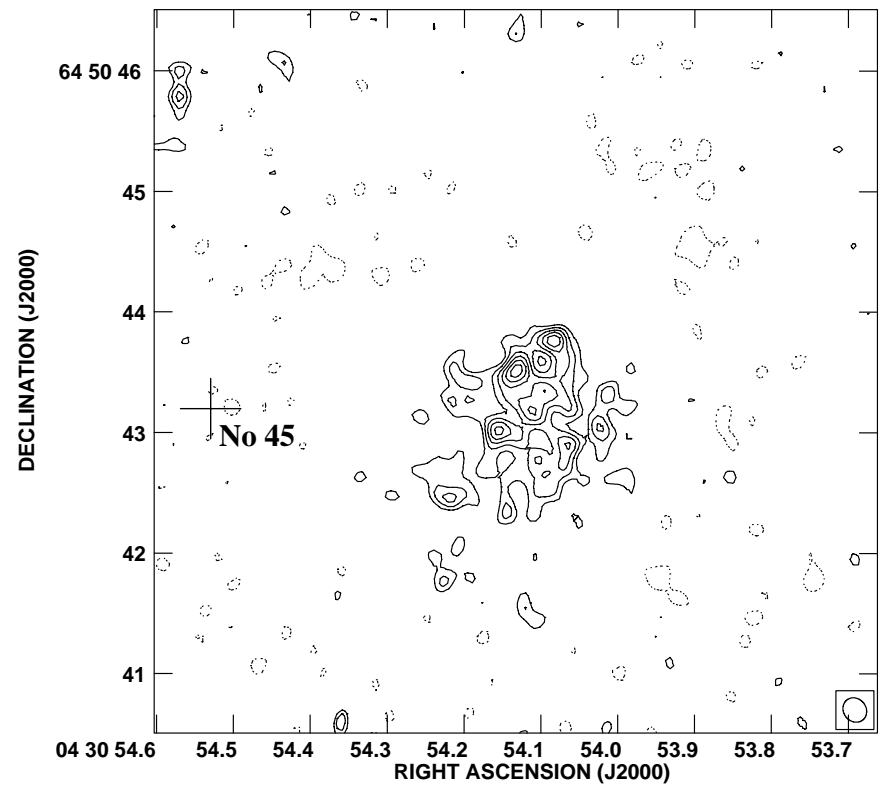

Fig. 7. 1.4 GHz MERLIN observation of source M-6 (Table 2) associated with the the $\mathrm{HII}$ regions 7 and 6 identified by Waller (1991). The contour interval is in steps of $30 \mu \mathrm{Jy} /$ beam with the first negative and positive contour at $50 \mu \mathrm{Jy} / \mathrm{beam}$ $\left(=2 \sigma_{\mathrm{n}}\right)$. The synthesized beam is shown in the lower right corner. The cross indicates an intermediate-sized stellar cluster No. 45 (Table 4).

\subsection{Non-thermal sources near star cluster $\mathrm{C}$ and the associated $\mathrm{CO}$ cloud}

The cluster $\mathbf{C}=$ No. 10 is associated with a bright H II region (No.2, Waller 1991) located at the edge of a CO cloud complex of $\sim 180 \mathrm{pc}$ extension and $1.4 \times 10^{6} \mathrm{M}_{\odot}$ total mass (Taylor et al. 1999, their Figs. 8 and 9). The sources M-1,2 (b,c,d), VLA-10 and VLA-11; the HII regions 1,2,3 (Waller 1991); and the CO clouds 1,2,3 (Taylor et al. 1999) form a structure which resembles the 30 Doradus (R 136) and N160 and N 159 region of the LMC (Cohen et al. 1988; Johansson et al. 1998; Bolatto et al. 2000), although less massive. Similar to the LMC, the dominant CO clouds 1,2, 3 (Taylor et al. 1999) are located at the Western end of NGC 1569's stellar bar (Waller \& Dracobly 1993) and extend from there in perpendicular direction to the major axis of the bar. Star formation has started at the Northern edge of the CO cloud, producing the cluster No. 10 (Hunter et al. 2000) and intermediatesize clusters. The structure around cluster $\mathbf{C}$ is difficult to assess; there are several tentative sources M-1, b, cd, but for VLA observations the region contains too much extended emission and confusion to allow a clear distinction and identification of sources. The source $\mathrm{M}-1$ is clearly a thermal source.

Star formation is probably still progressing towards the South into the CO cloud. There are two non-thermal sources, M-2 and VLA-11, at the edge and $\sim 20-30 \mathrm{pc}$ outside the CO cloud complex 1,2,3 (Taylor et al. 1999), respectively. If we interpret these sources as SNRs, or 
RSNe, we may conclude that at these positions half-way along and at the edge of the $\mathrm{CO}$ cloud, some star formation has taken place, or is currently taking place, although not (yet) very efficiently. This holds in particular for the source M-2 since its surrounding contains the young, intermediate-size star cluster No. 5 of Hunter et al. (2000).

There are two non-thermal sources to the North of cluster No. 10. The source VLA-16, at $\sim 25$ pc North, is probably a SNR, and possibly associated with the region of star cluster No.10 (C) or its immediate surrounding (Table 4). The same holds for the source M-3 which is associated with the cluster No. 8 of Hunter et al. (2000; Table 4). In this area lies also the tentative source M-a.

\subsection{The environment of the SSCs $A$ and $B$}

The SSC- $\mathbf{A}$ consists of two components (O'Connell et al. 1995; de Marchi et al. 1997; Hunter et al. 2000; see Table 4) and shows evidence of WR stars (GonzalezDelgado et al. 1997; Buckalew et al. 2000). Under the assumption of being a single object, Ho \& Filippenko (1996) derived a lower limit of the (dynamical) mass $M(\mathbf{A})=$ $(3.3 \pm 0.5) \times 10^{5} M_{\odot}$, based on the measured stellar velocity dispersion $\sigma_{*}=(15.7 \pm 1.5) \mathrm{km} \mathrm{s}^{-1}$ and the halflight radius of the cluster $r(\mathbf{A})=1.9 \pm 0.2 \mathrm{pc}\left(0.18^{\prime \prime}\right)$. Using the fact that $\mathbf{A}$ is double, de Marchi et al. (1997) and Sternberg (1998) obtain, for A1, a mass between $2.8 \times 10^{5} M_{\odot}$ and $1.1 \times 10^{6} M_{\odot}$. The $\operatorname{SSC}(\mathrm{s})-\mathbf{A}$ is located in a cavity, visible in $21 \mathrm{~cm}-\mathrm{H}$ I and X-ray emission as a $\sim 200$ pc diameter hole (Israel \& van Driel 1990; Heckman et al. 1995), The cavity is assumed to be blown out by SN explosions and stellar winds. The SSC- $\mathbf{B}$ is located in some diffuse interstellar material.

In Fig. 1, the circles around the clusters delineate areas of $\sim 50 \mathrm{pc}$ diameter. They represent, approximately, the distance a star escaping from a cluster with the velocity dispersion $\sigma_{*} \approx 20 \mathrm{~km} \mathrm{~s}^{-1}$ (Ho \& Filippenko 1996) can traverse within $\sim 1-2 \mathrm{Myr}$. The encircled fields delineate approximately the areas onto which a search for RSNe and SNRs, possibly associated with the SSCs, should concentrate. The areas should not be significantly larger since the SSCs (A) contain a large number of low mass stars and thus are likely to be gravitationally bound (Ho \& Filippenko 1996; Sternberg 1998; Smith \& Gallagher 2001) so that the probability that massive stars escape to larger distances is small, although the systems may not yet be fully relaxed.

The observations do not reveal a RSN or SNR in the immediate surrounding of the SSC $\mathbf{A}$ and $\mathbf{B}$, except for the source VLA- 8 assumed to be a SNR of $\sim 20$ pc diameter.

Besides the statistical argument brought forward to explain the absence of short-lived RSNe and RSNs in and near the SSCs, we believe that there exists also a valid kinematical argument for their absence. When extrapolating to SSCs Canto et al.'s (2000) calculation of the action of stellar winds of many massive stars in a cluster, combined with the action of several SN explosions, and when considering the influence of the cluster gravitational field on the propagation of the SN blast in a similar way as done for proto globular clusters (Shustov \& Wiebe 2000), a violent and turbulent outflow of hot material is expected to occur which leaves little room for a quiescent development of SNRs. Using the radius $r(\mathbf{A})$ and mass $M(\mathbf{A})$ of the SSC-A mentioned above, the stellar mass concentration $\rho$ and the average distance $<\delta>$ between the cluster stars is

$$
\rho \approx \frac{M(\mathbf{A})}{\left[4 / 3 \pi r(\mathbf{A})^{3}\right]} \approx \frac{3 \times 10^{5} M_{\odot}}{30 \mathrm{pc}^{3}} \approx 1 \times 10^{4} M_{\odot} \mathrm{pc}^{-3}
$$

and

$$
\langle\delta\rangle \propto(1 / \rho)^{1 / 3} \propto 0.05-0.1 \mathrm{pc},
$$

if we assume that the average mass of the cluster stars is $\sim 1-3 M_{\odot}$ (Sternberg 1998). Stellar winds and material ejected in SN explosions extend to similar distances which makes a strong interaction of cluster-internal gas plausible. An example of this process is SN 1993J in M 81 which has a shell diameter of $\sim 0.1 \mathrm{pc}$ some 1300 days after explosion and which expands with a velocity ${ }^{1}$ of $\sim 15000 \mathrm{~km} \mathrm{~s}^{-1}$ (Marcaide et al. 1997). The individual stellar winds and SN ejected hot material blow out of the SSC as a common wind, which diffuses through the interstellar medium. In the immediate surroundings of the SSCs the outflowing stellar winds and SN ejected material shock with the interstellar medium, forming shells and holes. The shock wave around SSC- $\mathbf{A}$ has probably created the $\mathrm{HI}$ hole and the shell seen in the observations of Israel \& van Driel (1990) and Greve et al. (1996). In this picture it is not surprising that the non-thermal source (VLA-8), which may be an extended SNR, is located $\sim 30 \mathrm{pc}$ outside the cluster core where the interstellar matter is in less turbulent motion. This picture of the diffusion of SN ejected gas agrees with the fact that locally metal-enriched gas has not been found (Kobulnicky \& Skillman 1997).

We do not find RSNe or SNRs in or near the many other star clusters (Hunter et al. 2000) and WR sources (Buckalew et al. 2000), respectively.

\subsection{A final remark}

The phenomenon of SSCs and SNRs is much more spectacular in the starburst galaxy M 82 than in the irregular galaxy NGC 1569; however, the heavy obscuration of M 82 prevents us from obtaining a complete view of the relation between SSCs and SNRs. The post-starburst galaxy NGC 1569 with locally recent star formation similar to that found in M82, allows on the other hand an unobscured view of many young stars in the body of the galaxy, of the large number of intermediate-size star clusters, and - as an exception to other unobscured closeby irregular galaxies - of two SSCs $\mathbf{A}$ and $\mathbf{B}$. The VLA detects

\footnotetext{
${ }^{1}$ In a dense (molecular cloud) gas, like in M 82, the expansion velocity can be significantly smaller (Chevalier \& Fransson 2001).
} 
Table 4. Positions of star clusters referred to in this study (Hunter et al. 2000; A1, A2: de Marchi et al. 1997).

\begin{tabular}{|c|c|c|c|c|c|c|}
\hline Cluster & $\begin{array}{l}\mathrm{RA}(2000) \\
{\left[\begin{array}{l}\mathrm{h} \mathrm{m} \mathrm{s} \\
\end{array}\right.}\end{array}$ & $\begin{array}{c}\operatorname{Dec}(2000) \\
{\left[\begin{array}{lll} & \prime & \prime \prime\end{array}\right]}\end{array}$ & $\begin{array}{c}M_{V}^{a} \\
{[\mathrm{mag}]}\end{array}$ & $\begin{array}{l}\operatorname{Radius}^{a} \\
{\left[{ }^{\prime \prime} \leftrightarrow \mathrm{pc}\right]}\end{array}$ & $\begin{array}{l}\text { Non-thermal } \\
\text { Source }\end{array}$ & $\begin{array}{c}\text { Distance of RSN, SNR } \\
\text { to Cluster [pc] }\end{array}$ \\
\hline $\mathrm{SSC}-\mathbf{A}$ & 43048.19 & 645058.6 & -14.1 & $1.14-12$ & [VLA-8: SNR & $\sim 30]$ \\
\hline $\mathrm{SSC}-\mathbf{A} \mathbf{1}^{b}$ & & & -13.6 & $\sim 0.15-1.6$ & & \\
\hline $\mathrm{SSC}-\mathbf{A} \mathbf{2}^{b}$ & & & -12.3 & $\sim 0.17-1.8$ & & \\
\hline SSC-B & 43048.99 & 645052.7 & -13.1 & $1.34-14$ & & \\
\hline C/No. 10 & 43047.26 & 645102.3 & -11.9 & $0.71-7.6$ & VLA-16: SNR & $\sim 25$ \\
\hline No. 5 & 43046.67 & 645054.4 & -8.6 & $0.46-4.9$ & $\mathrm{M}-2: \mathrm{RSN}^{c}$ & 15 \\
\hline No. 6 & 43046.89 & 645100.6 & -9.7 & $0.34-3.6$ & M-1: therm. source & \\
\hline No. 7 & 43046.96 & 645059.4 & -9.2 & $0.34-3.6$ & M-1: therm. source & \\
\hline No. 8 & 43047.04 & 645106.6 & -8.6 & $0.23-2.4$ & $\mathrm{M}-3: \mathrm{RSN}^{c}$ & 2 \\
\hline No. 18 & 43048.07 & 645057.3 & -7.8 & $0.18-1.9$ & VLA-8: SNR & 10 \\
\hline No. 45 & 43054.53 & 645043.2 & -6.9 & $0.50-6.0$ & M-6: SNR & 35 \\
\hline
\end{tabular}

${ }^{a}$ For a distance of $2.2 \mathrm{Mpc}$.

${ }^{b}$ Components of SSC-A, separated by 0.18" [2.2 pc]; A1 is located to the $\sim$ South-East of A2, Hunter et al. (2000).

${ }^{c}$ Or a small SNR.

the non-thermal sources VLA-8 and VLA-16 at $\sim 25 \mathrm{pc}$ distance from the SSC $\mathbf{A}$ and cluster $\mathbf{C}$, respectively. We did not detect these sources with MERLIN. We interpret these sources as low surface brightness SNRs. Because of their distance of $\sim 25 \mathrm{pc}$ from the clusters we hesitate to attribute their origin to stars originally belonging to these clusters. The regions closer to the SSCs and closer to the intermediate-size clusters are devoid of RSNe and SNRs. Although a comparison between M 82 and NGC 1569 on statistical arguments seems to provide a plausible explanation for the absence of SNRs near and in the clusters, some caution in the use of this argument is appropriate in view of the locally large number of stars $\left(10^{5}\right.$ to $\left.10^{6}\right)$ very recently formed in the SSCs and the intermediatesize clusters. The absence of SNRs in and very close to the clusters, in both M 82 and NGC 1569, may - at least partially - be due to the hostile environment. Unfortunately, within $\sim 5 \mathrm{Mpc}$ distance there are no other unobscured galaxies containing many SSCs, allowing a similar investigation with MERLIN.

Acknowledgements. We thank the MERLIN staff, Jodrell Bank, for the observations, the help in data reduction, and the pleasant hospitality. We thank the referee for putting the astrophysical question into the correct context of star formation and supernova explosions, and for eliminating contradictions.

\section{References}

Ables, H. D. 1971, Publ. US Naval Obs. XX, Part IV, 60 Aloisi, A., Clampin, M., Diolaiti, E., et al. 2001, AJ, 121, 1425 Arp, H. C., \& Sandage, A. 1985, AJ, 90, 1163

Bolatto, A. D., Jackson, J. M., Israel, F. P., et al. 2000, ApJ, 545,234

Buckalew, B.A., Dufour, R. J., Shopbell, P. L., \& Walter, D. K. 2000, AJ, 120, 2402

Canto, J., Raga, A. C., \& Rodriguez, L. F. 2000, ApJ, 536, 896 Chevalier, R. A., \& Fransson, C. 2001, ApJ, 558, L27

Clark, B. G. 1980, A\&A, 89, 377
Cohen, R. S., Dame, T. M., Garay, G., et al. 1988, ApJ, 331, L95

de Grijs, R., O’Connell, R. W., Becker, G. D., Chevalier, R. A., \& Gallagher, J. S. III 2000, AJ, 119, 681

de Grijs, R., O'Connell, R. W., \& Gallagher, J. S. III 2001, AJ, 121,768

de Marchi, G., Clampin, M., Greggio, L., et al. 1997, ApJ, 479, L27

Freedman, W., Hughes, S. M., Madore, B. F., et al. 1994, ApJ, 427,628

Gallagher, J. S. III, \& Smith, L. J. 1999, MNRAS, 304, 540

Golla, G., Allen, M. L., \& Kronberg, P. P. 1996, ApJ, 473, 244

Gonzalez-Delgado, R. M., Leitherer, C., Heckman, T. M., \& Cerviño, M. 1997, ApJ, 483, 705

Greggio, L., Tosi, M., Clampin, M., et al. 1998, ApJ, 504, 725

Greve, A., Becker, R., Johansson, L. E. B., \& McKeith, C. D. 1996, A\&A, 312, 391

Heckman, T. M., Dahlem, M., Lehnert, M. D., et al. 1995, ApJ, 448, 98

Ho, L. C., \& Filippenko, A. V. 1996, ApJ, 466, L83

Huang, Z. P., Thuan, T. X., Chevalier, R. A., Condon, J. J., \& Yin, Q. F. 1994, ApJ, 424, 114

Hunter, D. A., O'Connell, R. W., Gallagher, J. S., \& Smecker-Hane, T. A. 2000, AJ, 120, 2383

Israel, F. P. 1988, A\&A, 194, 24

Israel, F. P., \& de Bruyn, A. G. 1988, A\&A, 198, 109

Israel, F. P., \& van Driel, W. 1990, A\&A, 236, 323

Johansson, L. E. B., Greve, A., Booth, R. S., et al. 1998, 331, 857

Kobulnicky, H. A., \& Skillman, E. D. 1997, ApJ, 489, 636

Labrie, K., \& Pritchet, C. J. 1998, Proc. 5th CFHT User's Meeting, Quebec City, CA, May 1998, 147

Marcaide, J. M., Alberdi, A., Ros, E., et al. 1997, ApJ, 486, L 31

Mattila, S., \& Meikle, W. P. S. 2001, MNRAS, 324, 325

McLeod, K. K., Rieke, G. H., Rieke, M. J., \& Kelly, D. M. 1993, ApJ, 412, 111

Melnick, J., Moles, M., \& Terlevich, R. 1985, A\&A, 149, L24

Muxlow, T. W. B., Pedlar, A., Wilkinson, P. N., et al. 1994, MNRAS, 266, 455

O’Connell, R. W., \& Mangano, J. J. 1978, ApJ, 221, 62 
O'Connell, R. W., Gallagher, J. S. III, \& Hunter, D. A. 1994, ApJ, 433, 65

O'Connell, R. W., Gallagher, J. S. III, Hunter, D. A., \& Colley, W. N. 1995, ApJ, 446, L10

Origlia, L., Leitherer, C., Aloisi, A., et al. 2001, AJ, 122, 815

Pedlar, A., Muxlow, T. W. B., \& Garrett, M. 1999, MNRAS, 307,761

Prada, F., Greve, A., \& McKeith, C. D. 1994, A\&A, 288, 396

Sakai, S., \& Madore, B. F. 1999, ApJ, 526, 599

Seaquist, E. R., \& Bignell, R. C. 1976, A\&A, 48, 421

Shustov, B. M., \& Wiebe, D. S. 2000, MNRAS, 319, 1047

Smith, L. J., \& Gallagher, J. S. III 2001, MNRAS, 326, 1027

Sternberg, A. 1998, ApJ, 506, 721

Tarchi, A., Neininger, N., Greve, A., et al. 2000, A\&A, 358, 95
Taylor, C. L., Hüttemeister, S., Klein, U., \& Greve, A. 1999, A\&A, 349, 424

van der Hulst, J. M., de Grijs, R., \& Israel, F. P. 2001, in preparation (vdHGI)

Waller, W. 1991, ApJ, 370, 144

Waller, W. H., \& Dracobly, C. 1993, in Massive Stars: Their Lives in the Interstellar Medium, ed. J. Cassinelli, \& E. Churchwell, ASP Conf. Ser., 35, 492

Weiler, K. W., \& Sramek, R. A. 1988, ARA\&A, 26, 295

Wills, K. A., Pedlar, A., Muxlow, T. B. W., \& Wilkinson, P. N. 1997, MNRAS, 291, 517

Wills, K. A., Pedlar, A., \& Muxlow, T. B. W. 1998, MNRAS, 298,347 